\title{
Soft X-ray to far infrared luminosities ratio in star-forming galaxies: predictions from synthesis models
}

\author{
J. M. Mas-Hesse ${ }^{1,2}$, H. Otí-Floranes ${ }^{2,3}$, and M. Cerviño ${ }^{4}$ \\ 1 Centro de Astrobiología (CSIC-INTA), 28850 Torrejón de Ardoz, Spain \\ 2 Laboratorio de Astrofísica Espacial y Física Fundamental (LAEFF-INTA), POB 78, 28691 Villanueva de la Cañada, Spain \\ e-mail: mm@laeff.inta.es; otih@laeff.inta.es \\ 3 Dpto. de Física Moderna, Facultad de Ciencias, Universidad de Cantabria, 39005 Santander, Spain \\ ${ }^{4}$ Instituto de Astrofísica de Andalucía (CSIC), 18008 Granada, Spain \\ e-mail: mcs@iaa.es
}

Received 2 August 2007 / Accepted 15 January 2008

\section{ABSTRACT}

\begin{abstract}
Context. A good correlation has been found in star-forming galaxies, between the soft X-ray and the far infrared or radio luminosities. The soft X-ray emission in star-forming regions is driven by the heating of the diffuse interstellar medium, and by the mechanical energy released by stellar winds and supernova explosions, both directly linked to the strength of the star formation episode. Aims. We analyze the relation between the soft X-ray and far infrared luminosities as predicted by evolutionary population synthesis models, aiming first to test the validity of the soft X-ray luminosity as a star formation rate estimator, using the already known calibration of the FIR luminosity as a proxy, and second to propose a calibration based on the predictions of evolutionary synthesis models.

Methods. We have computed the soft X-ray and far infrared luminosities expected for a massive starburst as a function of evolutionary state, the efficiency of the conversion of mechanical energy into soft X-ray luminosity, the star formation history (instantaneous or extended) and dust abundance, and we have compared these predictions with observational values for 62 star-forming galaxies taken from the literature.

Results. The observational $L_{\mathrm{soft}} / L_{\mathrm{FIR}}$ ratios are consistent with the model predictions under realistic assumptions (young starbursts, and efficiency in the re-processing of mechanical energy of a few percent), confirming the correlation between the diffuse soft X-ray emission and the star formation episode.

Conclusions. The soft X-ray emission of the diffuse, extended gas surrounding massive star-forming regions, can be used as a star formation rate tracer. The empirical calibrations presented in the literature are supported by the predictions of evolutionary synthesis models, and by the analysis of a larger number of star-forming galaxies. The calibrations are, however, biased towards galaxies dominated by relatively unevolved starbursts.
\end{abstract}

Key words. ISM: kinematics and dynamics - ISM: supernova remnants - galaxies: starburst - X-rays: galaxies - X-rays: ISM

\section{Introduction}

The onset of massive star formation episodes in galaxies drives their observational properties in almost any wavelength range. The $U V$ and optical become dominated by the continuum of massive, hot and young stars, as well as by the presence of nebular emission lines. After a few Myr of evolution, red supergiant stars contribute to most of the near infrared emission. The heating of interstellar dust particles by the powerful $U V$ photons induces the thermal re-emission of large amounts of energy in the mid and far infrared domain. The injection of ionizing photons into the surrounding gas generates thermal radio emission, which is replaced by non-thermal emission as the ionizing power of the burst declines and the more massive stars begin to explode as supernovae. The direct relation between the strength of the star formation episode and the intensity of the different observable parameters has allowed a number of star formation rate calibrators to be defined, such as UV continuum, emission lines intensity, far infrared or radio luminosities (Kennicutt 1998; Rosa-González et al. 2002; Bell 2003). These calibrators have proven to be invaluable for statistical studies of the star formation history of the Universe.
Star-forming regions are also the source of conspicuous $\mathrm{X}$-ray emission, generated by individual stars, by the injection of large amounts of mechanical energy heating the interstellar medium, by supernova remnants, and by binary systems transferring mass to a compact primary (Cerviño et al. 2002; Persic \& Rephaeli 2002). All of these individual components are in principle directly linked to the strength of the star formation episode, so that the X-ray luminosity could also be used as an estimator of star formation rates (SFR).

Several authors have in recent years discussed the feasibility of using the X-ray luminosity as an SFR estimator. Fabbiano \& Shapley (2002) already concluded from the analysis of $234 \mathrm{~S} 0 / \mathrm{a}-$ Irr galaxies observed with Einstein, that the correlation they found between the X-ray and the FIR luminosities in Sc-Irr galaxies was due to the young stellar populations in these objects. Ranalli et al. (2003) proposed an empirical calibration of the soft $(0.5-2.0 \mathrm{keV})$ and hard $(2-10 \mathrm{keV}) \mathrm{X}$-ray luminosities, based on their correlation with the far infrared (FIR) and radio luminosities, and using the known calibrations of these parameters as proxies. Grimm et al. (2003) studied the correlation between the number of high-mass X-ray binaries (HMXB) and the $S F R$, deriving different calibrations of the hard X-ray 
luminosity for low and high star formation rates. Persic et al. (2004) obtained a calibration of the hard X-ray luminosity as a SFR estimator by assuming that most of the emission in this range is associated with $\mathrm{HMXB}$, and scaling from the number of HMXB to the SFR of our Galaxy. Gilfanov et al. (2004) confirmed the calibration of the hard X-ray luminosity associated with $\mathrm{HMXB}$, using slightly different slopes at high and low star formation rates. In a recent paper, Persic \& Rephaeli (2007) found indeed that the collective hard X-ray emission of young point sources correlates linearly with the star formation rate derived from the far infrared luminosity. Strickland et al. (2004a) demonstrated that the luminosity of diffuse X-ray emission in star-forming galaxies is directly proportional to the rate of mechanical energy injection from the young, massive stars into the interstellar medium of the host galaxies. A similar result was found by Grimes et al. (2005) from the analysis of a sample of starburst galaxies of different types (from dwarf starbursts to ultraluminous infrared galaxies), which concluded that the mechanism producing the diffuse X-ray emission in the different types of starbursts was powered by the mechanical energy injected by stellar winds and supernovae into the surrounding medium. Recently Rosa-González et al. (2007) confirmed the reliability of the soft X-ray luminosity as an SFR estimator from the analysis of a sample of star-forming galaxies in the Chandra Deep Field South at redshifts $z=0.01-0.67$, using the UV continuum luminosity from GALEX as a proxy.

While hard X-ray emission from star-forming regions may be dominated by binary systems, diffuse soft X-ray emission is generated by reprocessed, mechanical energy from stellar winds and supernovae explosions. This mechanical energy is related to the strength of the burst of star-formation, and can be calculated using evolutionary population synthesis models. In this paper, we analyze the correlation between the soft X-ray and FIR luminosities in star-forming regions, both predicted by evolutionary synthesis models. We study the $L_{\mathrm{SoftX}} / L_{\mathrm{FIR}}$ ratio as a function of the evolutionary state, the efficiency of the conversion of mechanical energy into soft X-ray luminosity, the star formation history (instantaneous or extended), and the dust abundance, and compare the computed values with observations taken from the literature. Our objective is to derive a calibration of $L_{\mathrm{soft}}$ as a tracer of the star formation rate, based on the predictions of evolutionary synthesis models, and to test the validity of the empirical calibration proposed by Ranalli et al. (2003).

In Sect. 2 we describe the evolutionary synthesis models that we use in the present study, in Sect. 3 we present the observational data taken from the literature, and in Sect. 4 we discuss the predictions and the comparison with the observational values. Throughout this work we have assumed $H_{0}=73 \mathrm{~km} \mathrm{~s}^{-1} \mathrm{Mpc}^{-1}$ to convert fluxes into luminosities.

\section{Evolutionary synthesis models}

We have computed the predicted $L_{\text {softX }}$ and $L_{\text {FIR }}$ values using the evolutionary population synthesis models of Cerviño et al. (2002), (hereafter CMHK02 models ${ }^{1}$ ), which are based on the models of Arnault et al. (1989), Mas-Hesse \& Kunth (1991) and Cerviño \& Mas-Hesse (1994). These models compute the evolution of a cluster of massive stars, formed at the same time (Instantaneous Bursts, IB), or during an extended period of time (typically several tens of Myr) at a constant rate (Extended Bursts, EB), assuming different metallicities and Initial Mass

1 Downloadable from http://www.laeff.inta.es/users/mcs/ SED/
Function (IMF) slopes. The CMHK02 models were developed to compute the evolution of a starburst during the first $30 \mathrm{Myr}$, following the onset of the star formation episode. Once the structure (number of stars of each spectral type and luminosity class at a given evolutionary time) of the stellar population is derived, the models are used to compute a number of observable parameters, from X-ray to radio wavelengths.

In general, we assume a solar metallicity $Z_{\odot}$, and a Salpeter $\operatorname{IMF}\left(\phi(m) \sim m^{-2.35}\right)$ with masses ranging between $2 M_{\odot}$ and $120 M_{\odot}$. The output of the models are normalized to the star formation rate (mass of gas transformed into stars per unit time, $M_{\odot} \mathrm{yr}^{-1}$ ) for EB cases, or star formation strength (mass of gas transformed into stars at the onset of the burst, in units of $M_{\odot}$ ) for IB scenarios. In both cases, the mass normalization corresponds to the mass integrated between $2 M_{\odot}$ and $120 M_{\odot}$ assuming a Salpeter IMF. We emphasize that this normalization might differ significantly if other mass limits are considered. For example, the ratio between our mass normalization $\left(2-120 M_{\odot}\right)$ and the one assumed by Kennicutt (1998) $\left(0.1-100 M_{\odot}\right)$ is $M_{2}^{120} / M_{0.1}^{100}=0.293$. It is important to remark that this is the normalization implicitly assumed when the SFR calibrations proposed by Kennicutt (1998) are used. Under our assumptions, the soft X-ray (0.4-2.4 keV) and far infrared luminosities were computed during the first $30 \mathrm{Myr}$ after the onset of the massive starburst episode.

One of the main sources of soft X-ray emission in a starforming region is the diffuse gas heated by mechanical energy from a starburst (massive stellar winds or supernova explosions) into the surrounding medium. Its contribution is modeled by a Raymond-Smith thermal plasma with $k T=0.5 \mathrm{keV}$, which controls the fraction of the mechanical energy that heats the gas to X-ray temperatures, $\epsilon_{\mathrm{xeff}}$. The models include the soft X-ray radiation emitted during the adiabatic phase of the Supernova Remnant (SNR), which is modeled by a composite RaymondSmith plasma with $k T=0.23,0.76$ and $1.29 \mathrm{keV}$. The total energy emitted by the SNR, during the adiabatic phase, has been subtracted from the energy of each supernova explosion when computing the injection of mechanical energy. A more detailed description of our models is provided in Cerviño et al. (2002).

The contribution of the stellar atmospheres to the soft X-ray emission was neglected because it is expected to be two orders of magnitude lower than the emission from the diffuse gas. The contribution of high-mass X-ray binaries to the soft X-ray emission has in addition been neglected in this work. Mas-Hesse \& Cerviño (1999) discussed the properties of the HMXB population expected to form during a massive star-formation burst. Binary systems become X-ray emitters when the primary collapses into a black hole or neutron star, the atmosphere of the secondary has started to expand, and the secondary is sufficiently close to the collapsed primary for mass transfer to begin. Mass is accreted onto the surface of the compact object and emits X-rays with a typical $L_{\mathrm{X}} \sim 10^{38} \mathrm{erg} \mathrm{s}^{-1}$, peaking at energies between 5 and $10 \mathrm{keV}$ (Persic \& Rephaeli 2002). The number of HMXB in a young starburst is dependent on many free parameters. Following Mas-Hesse \& Cerviño (1999), we estimate that only a few HMXB should be active after the first 5-6 Myr of evolution of starbursts that have transformed approximately $10^{6} M_{\odot}$ of gas into stars. This HMXB population should contribute a few times $10^{38} \mathrm{erg} \mathrm{s}^{-1}$ to the total X-ray luminosity, and a small fraction of this radiation to the soft X-ray emission. In all cases, the total contribution of $\mathrm{HMXB}$, to the soft X-ray emission, remains $15 \%$ for IB and $10 \%$ for EB. Only if one or a few of these HMXB develop into an ultraluminous X-ray source (ULX), with $L_{X} \sim 10^{40} \mathrm{erg} \mathrm{s}^{-1}$ (Miniutti et al. 2006), could the 
X-ray emission, from soft to hard X-rays, of the entire galaxy, be dominated by the emission of HMXB, compared to that of the diffuse gas.

Concerning the FIR emission, a thermal equilibrium of dust is assumed, implying that all the energy absorbed by dust, mostly originating from the UV continuum of the massive stars, is reemitted in the FIR range. In this paper, $L_{\mathrm{FIR}}$ refers to the total mid- and far-infrared luminosity integrated over the wavelength range $1-1000 \mu \mathrm{m}$. We remark that this parametrization of $L_{\mathrm{FIR}}$ implies a value of $L_{\mathrm{FIR}}$ that is larger than that calculated using the FIR parameter proposed by Helou et al. (1988), which is widely used in the literature. Helou et al. (1988) showed that the FIR luminosity computed from the IRAS fluxes at 60 and $100 \mu \mathrm{m}$ would intercept about $70 \%$ ( $0.15 \mathrm{dex})$ of the total FIR luminosity from 1 to $1000 \mu \mathrm{m}$, assuming that there is a single, dominating component, at a temperature of 30 to $50 \mathrm{~K}$. The discrepancy would be even larger in the presence of an additional warm dust component. This estimate of $L_{\mathrm{FIR}}$ is consistent with the range (8-1000 $\mu \mathrm{m})$ considered by Kennicutt (1998) for the calibration of $L_{\mathrm{FIR}}$ as an $S F R$ estimator, since most of the FIR luminosity in starburst galaxies is emitted at wavelengths in the range 10$120 \mu \mathrm{m}$. The models apply a Galactic extinction law (Cardelli et al. 1989) to the synthetic spectral energy distributions, which is parametrized by the colour excess $E(B-V)$. The energy absorbed due to extinction is calculated using the models. It is assumed that all energy absorbed is reemitted thermally by the dust, within the mid to far infrared domain, i.e., within the $\sim 8-$ $1000 \mu \mathrm{m}$ range. The models do not predict the shape of the FIR emission, since we do not make any assumption about the expected dust temperature. The presence of completely-obscured stars is not taken into account. Furthermore, the models assume that a fraction $1-f$ of Lyman continuum photons is directly absorbed by the dust, and does not contribute to the ionization (see Mezger et al. 1974). Mezger (1978) for the Galactic and Degioia-Eastwood (1992) for the Large Magellanic Cloud HII regions, derived $1-f$ values in the range $0.3-0.4$. We have assumed $(1-f)=0.3$ in this work, as proposed for starburst galaxies by Belfort et al. (1987).

The evolution of both $L_{\mathrm{FIR}}$ and $L_{\mathrm{soft}}$ predicted by the models are shown in Fig. 1, while their ratio $L_{\mathrm{softX}} / L_{\mathrm{FIR}}$ is presented in Fig. 2. For IB models, the luminosities are shown scaled to $1 M_{\odot}$ of gas transformed into stars at the onset of the starburst. In the case of EB models, the luminosities are normalized to $S F R=1 M_{\odot} \mathrm{yr}^{-1} . L_{\mathrm{FIR}}$ is presented in the plot, computed for $E(B-V)=0.1,0.5$ and 1.0. It can be seen that FIR emission saturates rapidly for $E(B-V)$ values above 0.5 . In the remainder of this work, we consider the value of $L_{\mathrm{FIR}}$ calculated by assuming that $E(B-V)=1.0$, which can be considered an upper limit to the expected FIR luminosity. In the case of EB models, $L_{\text {FIR }}$ reaches an asymptotic value after approximately 10-15 Myr of evolution, when an equilibrium is reached between the number of massive stars that die, and those forming continuously. For coeval starbursts, $L_{\mathrm{FIR}}$ declines rapidly after the first $5 \mathrm{Myr}$ of evolution, when the most massive stars begin to end their lifetimes and stop heating the interstellar dust.

$L_{\text {softX }}$ is shown in Fig. 1 computed for $\epsilon_{\text {xeff }}$ values of $1 \%$, $5 \%$ and $10 \%$. During the first few Myr, there is a rapid increase in $L_{\text {softx }}$ because both the luminosity and the stellar winds of the most massive stars, increase. After the first 3 Myr of evolution, the most massive stars end their lifetimes and explode as supernovae. The injection of mechanical energy begins to be dominated by energy released by supernovae explosions, as the importance of stellar winds rapidly diminishes. During the first $35 \mathrm{Myr}$, this remains the situation for IB models because, for a
Salpeter IMF, the supernovae rate declines slowly, while there exist stars of sufficient mass (of initial mass above $8 M_{\odot}$ ) to produce supernovae (Cerviño \& Mas-Hesse 1994; Leitherer \& Heckman 1995). In the case of EB models, $L_{\mathrm{soft}}$ is expected to increase slowly after the first $5 \mathrm{Myr}$, until an equilibrium is reached between the formation and destruction of stars that end their lifes as supernovae, i.e., at around $40 \mathrm{Myr}$ at solar metallicities, according to the evolutionary tracks considered. Leitherer \& Heckman (1995) presented the evolution of both supernova rate and the injection rate of mechanical energy for longer term evolution, up to ages of 300 Myr. Their Fig. 56 showed that the asymptotic rate of energy injection is within 0.05 dex of the value predicted at $30 \mathrm{Myr}$. Although the computation of the soft X-ray luminosities in the CMHK02 models is simplistic, the predictions are in good agreement with the results of Strickland \& Stevens (1999), which were computed using hydrodynamical simulations of a young superbubble driven by a cluster of massive stars.

Figure 2 shows the predictions for the $L_{\mathrm{SoftX}} / L_{\mathrm{FIR}}$ ratio evolution with time. It can be seen that $L_{\mathrm{softX}} / L_{\mathrm{FIR}}$ increases continuously with time after an instantaneous burst, even after the first $5 \mathrm{Myr}$, due to the fact that $L_{\mathrm{soft}}$ remains almost constant while $L_{\text {FIR }}$ decreases rapidly. In extended burst models, the rate of increase of $L_{\mathrm{SoftX}} / L_{\mathrm{FIR}}$ with time, after the first $5 \mathrm{Myr}$, is smaller. As discussed above, we expect the $L_{\mathrm{softX}} / L_{\mathrm{FIR}}$ ratio to stabilize after about $40 \mathrm{Myr}$ in EB models, when an equilibrium between formation and destruction of stars susceptible to becoming supernovae, has been reached. In both cases, there is a rapid increase in the $L_{\mathrm{softX}} / L_{\mathrm{FIR}}$ ratio after the onset of the burst (one order of magnitude in $3 \mathrm{Myr}$ in IB models), associated with the rapid increase in the amount of mechanical energy injected during the first phase of the evolution of the most massive stars.

In Fig. 3, we plot the evolution of the $L_{\mathrm{softX}} / L_{\mathrm{FIR}}$ ratio as a function of metallicity $\left(Z=Z_{\odot}\right.$ and $\left.Z=0.4 \times Z_{\odot}\right)$ and star formation history (instantaneous and extended bursts). Varying metallicity highlights two results: first, low-metallicity stars evolve more slowly and have a longer lifetime, such that the evolution of $L_{\mathrm{FIR}}$ is delayed with respect to solar-metallicity stars. Second, the lower the metallicity, the less efficient are the stellar winds, and therefore the lower is the amount of mechanical energy released into the interstellar medium. As seen in Fig. 3, the net effect is a decrease in the value of $L_{\text {softX }} / L_{\text {FIR }}$ at intermediate ages by 0.2 dex, or by up to 0.5 dex within the first $4 \mathrm{Myr}$ of evolution. At some ages, the trend is even reversed. For the time interval considered, however, the predicted values of $L_{\mathrm{softX}} / L_{\mathrm{FIR}}$ are similar for both values of metallicity.

\section{Observational data sample}

We have compiled $L_{\mathrm{softX}}$ and $L_{\mathrm{FIR}}$ data for 62 star-forming galaxies, of different types and redshifts, to compare with our model predictions.

The data compilation of Ranalli et al. (2003, hereafter $\mathrm{RCS03}$ ) is for star-forming galaxies, from the atlas of Ho et al. (1997), that have detectable X-ray emission in ASCA and/or BeppoSAX observations. Only spiral and irregular galaxies from Sa to later types were included in this sample, which was complemented by the authors with data of six, well-known starburst galaxies observed in the southern hemisphere.

The sample compiled by Tüllmann et al. (2006b, hereafter TUL06) is based on the nine late-type starburst galaxies of Tüllmann et al. (2006a) (XMM-Newton data), seven star-forming disk galaxies from Strickland et al. (2004b) (Chandra data) and seven additional late-type star-forming galaxies taken from the 

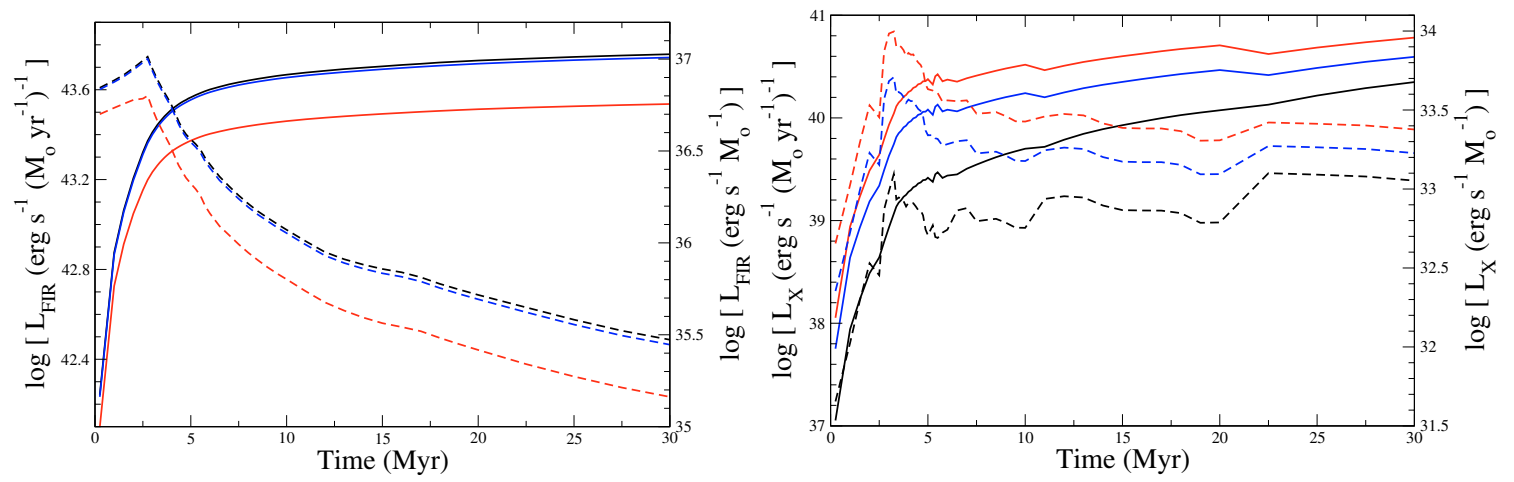

Fig. 1. Evolution of $L_{\mathrm{FIR}}(l e f t)$ and $L_{\mathrm{softX}}(r i g h t)$ for IB (dashed line, right axis) and EB (solid line, left axis) models. IB models predictions are shown normalized to $1 M_{\odot}$ of gas transformed into stars, while for EB the luminosities are scaled to $S F R=1 M_{\odot} \mathrm{yr}^{-1}$. $L_{\mathrm{FIR}}$ has been plotted (from top to bottom) for $E(B-V)=1.0,0.5$ and 0.1 . $L_{\text {softX }}$ has been computed (from top to bottom) for $\epsilon_{\mathrm{xeff}}=0.1,0.05$ and 0.01 .
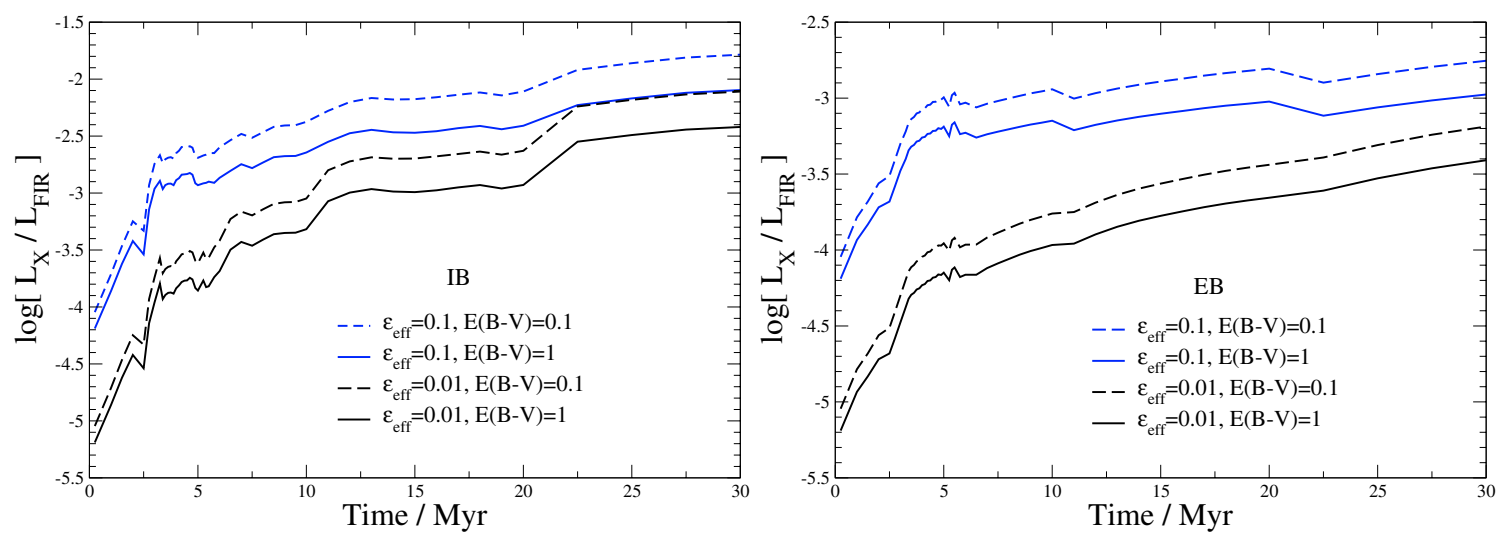

Fig. 2. Evolution of the $L_{\mathrm{soft} X} / L_{\mathrm{FIR}}$ ratio computed for $\epsilon_{\mathrm{xeff}}=0.01,0.1$ and $E(B-V)=0.1,1.0$. Left: predictions for IB models; right: predictions for EB models.

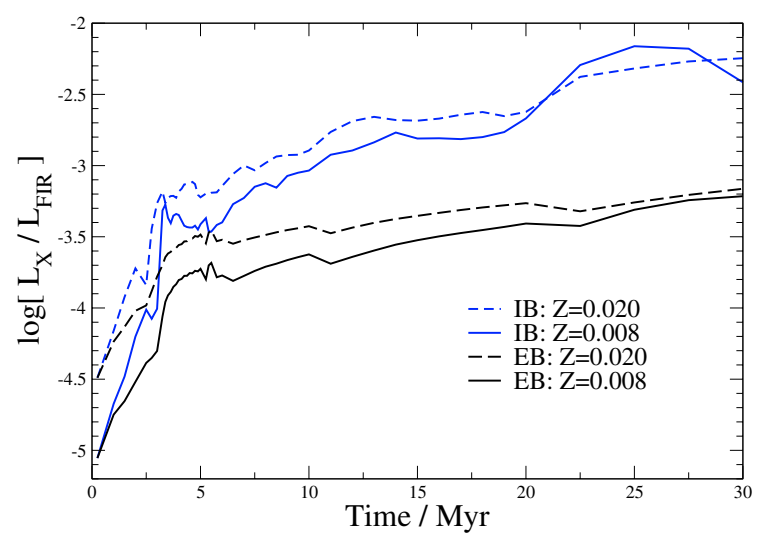

Fig. 3. Evolution of the $L_{\mathrm{soft}} / L_{\mathrm{FIR}}$ ratio as a function of metallicity and star formation regime. $E(B-V)=1.0$ and $\epsilon_{\text {xeff }}=0.05$.

literature. The contribution by obvious point-sources within the extraction regions was removed from all objects in the original Tüllmann et al. (2006a) and Strickland et al. (2004b) compilations when possible.

In both samples, the X-ray emission was corrected for Galactic neutral Hydrogen absorption, but not for the intrinsic absorption of the galaxies. The published far-infrared fluxes were computed using the IRAS 60 and $100 \mu \mathrm{m}$ flux following the procedure of Helou et al. (1988), corresponding to the energy emitted within the range 40-120 $\mu \mathrm{m}$. Calzetti et al. (2000) found for local starburst galaxies that $F(1-1000) / F(40-$ $120)=1.75 \pm 0.25$. We have therefore multiplied the FIR fluxes provided by RCS03 and TUL06 by 1.75 , in order to obtain a more realistic determination of the total amount of energy being reemitted in the mid- and far- infrared range. The luminosities were recomputed for all objects from the published fluxes by using the distances corrected to the Local Group reference frame as given in the Nasa Extragalactic Database, assuming $H_{0}=73 \mathrm{~km} \mathrm{~s}^{-1} \mathrm{Mpc}^{-1}$.

There is some overlap between the two samples. The $L_{\text {softX }} / L_{\text {FIR }}$ ratio for $\mathrm{M} 82$ is -4.08 in $\mathrm{RCS03}$ (based on BeppoSAX data) and -3.85 for TUL06 (Chandra). For NGC $4631 L_{\text {softX }} / L_{\text {FIR }}$ varies between -3.96 (ASCA) and -4.17 (XMM-Newton), respectively. In these cases we have taken the $L_{\text {softX }}$ values provided by TUL06 .

To compare with star-forming galaxies outside the Local Universe, we consider galaxies at redshifts $z=0.01-0.67$ studied by Rosa-González et al. (2007, hereafter ROSA07). Both $L_{\text {softX }}$ and $L_{\text {FIR }}$ data are available for these galaxies, which were observed as part of the Chandra Deep Field South (CDFS) survey. The X-ray data in this sample come from the cata$\log$ of Alexander et al. (2003), and were not corrected for either Galactic or intrinsic absorption by neutral hydrogen. Nevertheless, along the line of sight to the CDFS the expected Galactic neutral hydrogen absorption in the soft X-rays band is only $4.2 \%$ (0.02 dex) (Alexander et al. 2003). We exclude objects believed to harbour an obscured AGN, or to be dominated by low-mass, X-ray binaries (LMXB). In total, we have identified 18 objects with detectable soft X-ray, and far-infrared fluxes that appear not to be contaminated by either an AGN or LMXB. 


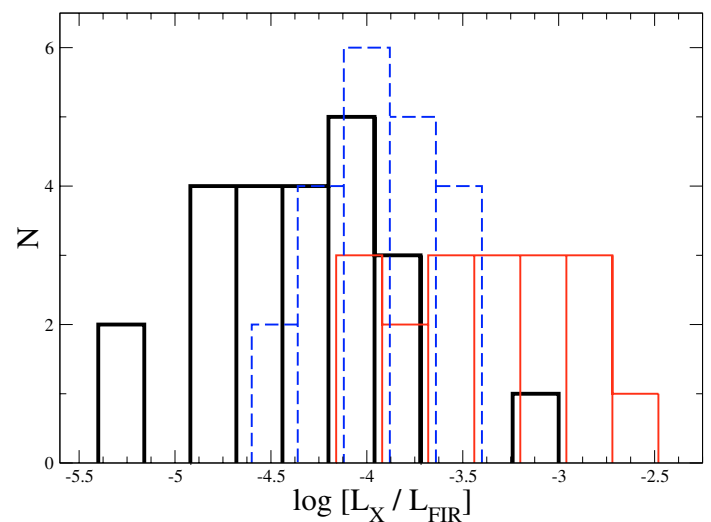

Fig. 4. $L_{\mathrm{soft}} / L_{\mathrm{FIR}}$ histograms for the samples of star-forming galaxies from Ranalli et al. (2003) (dashed line), Tüllmann et al. (2006b) (thick solid line) and Rosa-González et al. (2007) (thin solid line). The bins have been computed with a slight shift between each other for clarity.

ROSA07 derived the far infrared luminosities for these objects in the full $8-1000 \mu \mathrm{m}$ band using Spitzer observations at $25 \mu \mathrm{m}$. They used the empirical calibration of Takeuchi et al. (2005) based on the analysis of a large sample of galaxies for which fluxes in the four IRAS bands at 12, 25, 60 and $100 \mu \mathrm{m}$ were available. The FIR luminosity computed in this way should be consistent with the luminosities derived for the RCS03 and TUL06 samples, extrapolated to the $1-1000 \mu \mathrm{m}$ range. ROSA07 assumed $H_{0}=70 \mathrm{~km} \mathrm{~s}^{-1} \mathrm{Mpc}^{-1}$ to convert fluxes into luminosities.

We note that the $L_{\mathrm{softX}}$ values are integrated over the $0.5-2.0 \mathrm{keV}$ band by RCS03, $0.2-2.0$ by ROSA07, and over the $0.3-2.0 \mathrm{keV}$ band by TUL06, while the model predictions were computed for the $0.4-2.4 \mathrm{keV}$ band. We have verified that for the typical spectral properties of the diffuse gas in these objects, the discrepancies associated with the different bandwidths should be smaller than $9 \%$ (0.04 dex) in any case.

We have plotted in Fig. 4 the histograms of the $L_{\mathrm{softX}} / L_{\mathrm{FIR}}$ distribution for each sample. It can be seen that while there is a significant overlap between them, the star-forming galaxies compiled by RCS03 show the smallest dispersion. The TUL06 galaxies show generally lower $L_{\mathrm{softX}} / L_{\mathrm{FIR}}$ values than the objects compiled by RCS03, while the sample of ROSA07 presents the highest $L_{\mathrm{soft}} / L_{\mathrm{FIR}}$ ratios. The mean $\log \left(L_{\mathrm{softX}} / L_{\mathrm{FIR}}\right)$ values derived for each sample are -3.94 (0.27) (RCS03), -4.34 (0.48) (TUL06), -3.37 (0.45) (ROSA07) and -3.92 (0.57) for the whole compilation. Values within parentheses correspond to the $\sigma$ dispersion of each sample. We have looked for any possible correlation between the $L_{\mathrm{soft}} / L_{\mathrm{FIR}}$ ratios in the TUL06 galaxies and their morphological type, but there is no clear trend. Both $\mathrm{Sc}+\mathrm{Sd}$ and SB galaxies in the sample cover a wide range of luminosities and $L_{\mathrm{softX}} / L_{\mathrm{FIR}}$ values and furthermore, they cover a similar range in luminosities than the galaxies in the RCS03 sample. There is no obvious reason for the differences between the two samples of local star-forming galaxies.

\section{Discussion}

Figure 2 indicates that our models predict a strong dependence of the $L_{\text {softX }} / L_{\text {FIR }}$ ratio on the star formation history (instantaneous or extended), and on the evolutionary state of the star formation process. Moreover, the ratio is also strongly dependent on the

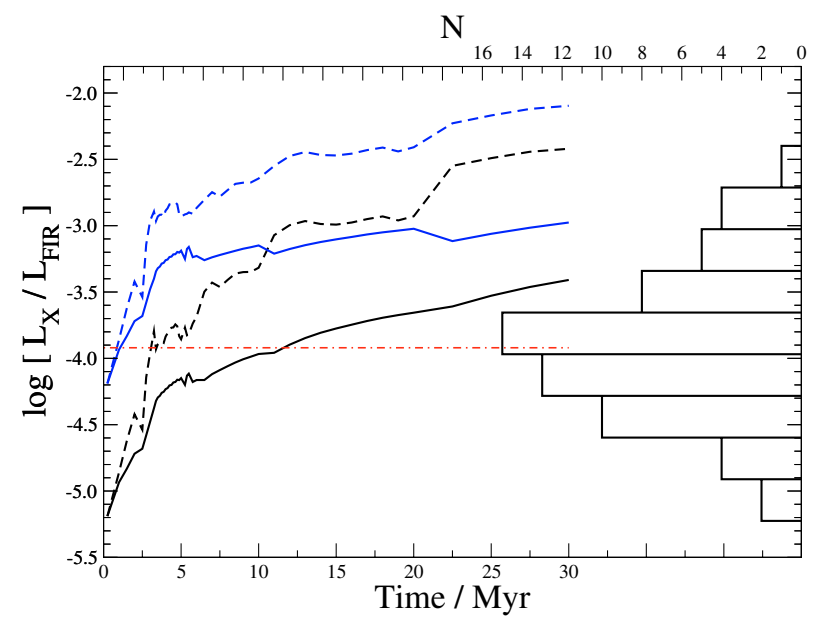

Fig. 5. $L_{\mathrm{softX}} / L_{\mathrm{FIR}}$ histogram for the combined sample, over the evolution of the $L_{\mathrm{soft}} / L_{\mathrm{FIR}}$ computed for IB (dashed lines) and EB (solid lines) and $\epsilon_{\mathrm{xeff}}=0.10$ (top) and 0.01 (bottom). The horizontal line corresponds to the mean ratio of the complete sample, $\log \left(L_{\mathrm{softX}} / L_{\mathrm{FIR}}\right)=$ -3.92 .

efficiency of the reprocessing of mechanical energy and $U V$ photons into soft X-ray and FIR emission, respectively. Therefore, we would expect a significant scatter in the $L_{\mathrm{soft}} / L_{\mathrm{FIR}}$ values observed in star-forming galaxies. This is indeed what we find in Fig. 4, as discussed above.

Mas-Hesse \& Kunth (1999) showed that the star formation episodes taking place in compact starburst or HII galaxies are generally of short duration, and that their properties can be better reproduced by evolutionary synthesis models assuming (nearly) instantaneous bursts, than by long-lasting, extendedin-time, star-formation processes. The generally strong optical emission lines in these galaxies constrain the evolutionary state of their massive star-formation episodes to ages below $6 \mathrm{Myr}$, typically within 4-5 Myr. On the other hand, star formation is expected to proceed during long periods of time in the disks of late-type spiral galaxies, generally in the form of individual bursts at different times. The accumulation of individual starburst episodes along the disks of these galaxies mimick a continuous star formation process (Mas-Hesse \& Kunth 1999), so that extended star formation models might be a better approximation to reproduce their spatially-integrated properties.

In Fig. 5, we have added to the predictions of the $L_{\mathrm{softX}} / L_{\mathrm{FIR}}$ values the histogram corresponding to the whole sample, as well as the average observational $L_{\mathrm{soft}} / L_{\mathrm{FIR}}$ value. As discussed above, there is a degeneracy between $\epsilon_{\mathrm{x} e f f}$ and the age of the star formation episode, so that it is not possible to discriminate between both parameters without additional constraints on the evolutionary state. Apart from a few irregular galaxies, for which an instantaneous model would describe better the star-formation episodes they are hosting, most of the galaxies in the sample are large spiral galaxies experiencing long-term star formation. Figure 5 shows that the mean $L_{\mathrm{softX}} / L_{\mathrm{FIR}}$ ratio can be reproduced by relatively young EB models (after about $10 \mathrm{Myr}$ of evolution) with moderate efficiencies $\epsilon_{\mathrm{x} e f f}$ of about $1 \%$. Galaxies with higher $L_{\text {softX }} / L_{\text {FIR }}$ ratios would correspond to more evolved extended starbursts reaching the evolutionary asymptotic phase, with $\epsilon_{\mathrm{x} e f f}$ in most cases below $10 \%$. On the other hand, the galaxies with lower $L_{\mathrm{softX}} / L_{\mathrm{FIR}}$ values can only be reproduced by very young models, after less than $10 \mathrm{Myr}$ of evolution. The galaxies in the sample have been selected in the various compilations for being star-forming or starburst galaxies, i.e., galaxies 
experiencing a stronger than average episode of star formation. The integrated emission in the far infrared and soft X-ray bands of some of these galaxies could be dominated by a single but intense burst of star formation. While star formation proceeds in these objects for a long time, these individual, intense bursts are not expected to last longer than a few Myr. Some of these starbursts could indeed be rather unevolved. We believe that this is what we see in Fig. 5: some of the galaxies with low $L_{\mathrm{softX}} / L_{\mathrm{FIR}}$ ratios could be dominated by a single, intense and relatively unevolved burst of star formation. A deeper study of the individual galaxies would be required to confirm this hypothesis, but it is beyond the scope of this work.

Grimes et al. (2005) computed the $L_{\text {softX }} / L_{\text {FIR }}$ ratios for a sample of ultraluminous infrared, starburst and dwarf starburst galaxies (the galaxies classified as starburst are already included in the TUL06 sample). For their 7 dwarf starburst galaxies (2 are already included in the sample by RCS03), they derived a mean $\log \left(L_{\mathrm{softX}} / L_{\mathrm{FIR}}\right)$ of -4.0 , close to the average value found for our complete compilation. The $L_{\mathrm{Soft}} / L_{\mathrm{FIR}}$ ratio of these galaxies (within the range -3.58 to -4.29 ) is properly reproduced by IB models at approximately 4-5 Myr, with $\epsilon_{\text {xeff }}$ within a realistic range 1-5\% (Strickland \& Stevens 1999; Summers et al. 2001, 2004). The mean $\log \left(L_{\mathrm{soft} X} / L_{\mathrm{FIR}}\right)$ value of the 9 ultraluminous infrared galaxies (ULIRG) in their sample is -4.5 , clearly below our average. This indicates that the star formation processes in this kind of galaxy might be relatively unevolved and their emission dominated by a single, intense episode of star formation.

In Fig. 6, we plot the observational $L_{\text {softX }}$ vs. $L_{\text {FIR }}$ values for the galaxies in the three samples. We include the predictions of IB and EB models at different ages, and for $\epsilon_{\mathrm{xeff}}$ values between 1 and $10 \%$. These plots support the main points of our previous discussion: the observational $L_{\mathrm{softX}}$ vs. $L_{\mathrm{FIR}}$ correlation in starforming galaxies can be well-reproduced by evolutionary synthesis models, assuming realistic parameters: age below $6 \mathrm{Myr}$ for IB cases, and a spread of young to evolved bursts for EB models, a high efficiency in the reprocessing of $U V$ photons into far infrared emission, and a moderate (1-10\%) efficiency in the heating of the diffuse interstellar gas by the mechanical energy released by massive stellar winds.

We have analyzed the effect of some intrinsic properties of the sampled objects, on the dispersion shown in the correlation plots. First, as noted above, the measured $L_{\text {softx }}$ values did not include the correction for intrinsic neutral hydrogen absorption. Kunth et al. (1998) measured the column density of neutral hydrogen in the line of sight to 8 compact starburst galaxies, by fitting their Lyman $\alpha$ profiles, finding values in the range $\log \left(n_{\mathrm{H}}\right) \sim 19-21 \mathrm{~cm}^{-2}$. We have computed that for the typical spectral properties of the hot diffuse gas this correction would be within $0.4 \%-50 \%$ (i.e. $<0.18 \mathrm{dex}$ ). A second effect would be the contamination of the soft X-ray emission by low mass $\mathrm{X}$-ray binaries associated to the underlying, older stellar population. ROSA07 estimated that the contribution by LMXB in their sample of spiral star-forming galaxies was generally of few percent, and concluded that the contamination should be negligible for galaxies with $S F R>1 M_{\odot} \mathrm{yr}^{-1}$. In the RCS03 sample only 7 objects have a value of $S F R$ below $1 M_{\odot} \mathrm{yr}^{-1}$, but their average $\log \left(L_{\mathrm{soft}} / L_{\mathrm{FIR}}\right)$ is -3.7 (in any case within -4.2 to -3.2$)$, i.e., with no deviation at all from the total average $L_{\mathrm{SoftX}} / L_{\mathrm{FIR}}$ ratio. Therefore, the contamination of $L_{\mathrm{soft}}$ by LMXB does not seem to be important. Finally, TUL06 removed the contamination by point sources before computing the integrated $L_{\mathrm{softX}}$, so that the contamination of this sample by LMXB should be negligible. An additional effect is related to the relative strength of the starburst emission compared to the galaxy as a whole. While the soft
X-ray emission would be associated mostly with the starburst regions, the far infrared luminosity can include a significant contribution from the rest of the galaxy, so that the $L_{\text {softX }} / L_{\text {FIR }}$ ratios obtained from spatially-integrated measurements would be lower than the intrinsic value produced by the starburst itself. A detailed morphological analysis of the galaxies in the sample is out of the scope of this paper, but we expect that at least in some cases the observational $L_{\mathrm{soft}} / L_{\mathrm{FIR}}$ value might be contaminated by emission not related to the star formation episode.

Finally, $L_{\mathrm{FIR}}$ has been computed assuming almost complete reprocessing of UV stellar continuum photons $(E(B-V)=1)$, as discussed in Sect. 2. Lower values of the extinction, of the order of $E(B-V)=0.1$, would raise the predicted $L_{\mathrm{soft}} / L_{\mathrm{FIR}}$ ratios by up to 0.15 dex. The effective extinction of these objects should be in between both extreme values of $E(B-V)$. In conclusion, correcting the soft X-ray luminosity from intrinsic photoelectric absorption and/or rejecting the far infrared emission not associated with the starburst regions themselves, could increase the $L_{\text {softX }} / L_{\text {FIR }}$ ratios for some objects. On the other hand, smaller interstellar extinction values would increase the predicted $L_{\text {softx }} / L_{\text {FIR }}$ ratio by less than $40 \%$.

We consider if it is possible to derive a calibration that would allow the $L_{\text {softx }}$ luminosity to be used as a tracer of SFR. Strickland \& Stevens (1999) found from detailed hydrodynamical simulations that superbubbles accelerated by the release of mechanical energy in a starburst, would convert on average approximately $5 \%$ of the input mechanical energy into soft X-ray emission. Summers et al. (2001) analyzed Mrk 33, a dwarf starforming galaxy, and concluded that it is dominated by an intense burst 5-6 Myr old, and that the rate of injection of mechanical energy from the starburst is approximately $1.2 \times 10^{41} \mathrm{erg} \mathrm{s}^{-1}$. The soft X-ray luminosity of the central, extended diffuse gas derived by these authors is $L_{\mathrm{soft}} \sim 2.2 \times 10^{39} \mathrm{erg} \mathrm{s}^{-1}$, corresponding to $\epsilon_{\mathrm{xeff}} \sim 0.018$. Similarly, Summers et al. (2004) estimated the mechanical energy injection rate from the starburst in NGC 5253 to be $L_{\text {mech }}=3.6 \times 10^{40} \mathrm{erg} \mathrm{s}^{-1}$. The measured thermal X-ray emission associated with the starburst was $L_{\mathrm{softX}} \sim 4 \times 10^{38} \mathrm{erg} \mathrm{s}^{-1}$, yielding $\epsilon_{\mathrm{xeff}} \sim 0.01$.

We can therefore assume that $\epsilon_{\text {xeff }}$ is constrained to be in the range $1-5 \%$ for typical star-forming galaxies. Our evolutionary synthesis models predict for Instantaneous Bursts (of age between 3-6 Myr) that $\log \left(L_{\mathrm{SoftX}} / L_{\mathrm{FIR}}\right)$ is approximately equal to $\log \left(L_{\mathrm{softX}} / L_{\mathrm{FIR}}\right) \sim(-3.1,-4.0)$, with a central value $\log \left(L_{\mathrm{softX}} / L_{\mathrm{FIR}}\right) \sim-3.5$. On the other hand, Extended Burst models predict $\log \left(L_{\mathrm{soft}} / L_{\mathrm{FIR}}\right) \sim(-3.0,-3.4)$ after $30 \mathrm{Myr}$ of evolution, when the number of supernova explosions begins to stabilize. For less-evolved, extended episodes (of approximately $10 \mathrm{Myr}$ of continuous star formation) the predicted ratios would be lower, within the range $\log \left(L_{\mathrm{Soft}} / L_{\mathrm{FIR}}\right) \sim(-3.5,-4.0)$.

These values are close to the average ratio $\log \left(L_{\mathrm{soft}} / L_{\mathrm{FIR}}\right) \sim$ -3.92 derived from our total sample of star-forming galaxies, supporting the use of the soft X-ray emission as a tracer of the star formation rate in starburst galaxies. Using the $L_{\text {softX }}$ values predicted by the models, as shown in Fig. 1, we can derive the calibration of the star formation rate (or strength) as a function of $L_{\mathrm{softX}}$. For this we have considered the points at which $\log \left(L_{\mathrm{softX}} / L_{\mathrm{FIR}}\right)=-3.5$ for IB models, $\log \left(L_{\mathrm{softX}} / L_{\mathrm{FIR}}\right)$ $=-3.1$ for $\mathrm{EB}$ cases in the asymptotic phase of evolution, and $\log \left(L_{\mathrm{softX}} / L_{\mathrm{FIR}}\right)=-3.7$ for extended, but non-evolved bursts, with ages of approximately $10 \mathrm{Myr}$, according to the discussion above. The calibrations would therefore be:

$$
\begin{aligned}
& \operatorname{SFR}\left(M_{\odot} \mathrm{yr}^{-1}\right)=2 \times 10^{-41} L_{\mathrm{softX}}\left(\mathrm{erg} \mathrm{s}^{-1}\right)(\text { evolved EB)} \\
& \operatorname{SFR}\left(M_{\odot} \mathrm{yr}^{-1}\right)=8 \times 10^{-41} L_{\mathrm{softX}}\left(\mathrm{erg} \mathrm{s}^{-1}\right)(\text { young EB}) .
\end{aligned}
$$



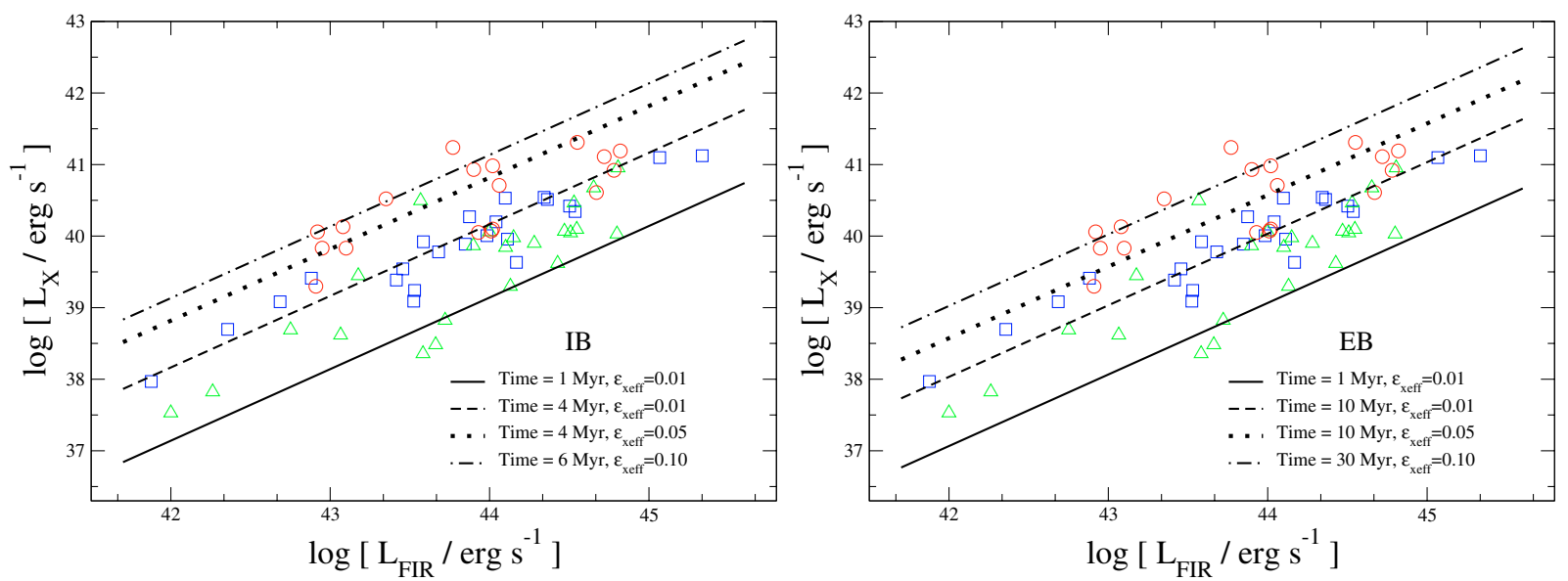

Fig. 6. $L_{\text {softX }}$ vs. $L_{\mathrm{FIR}}$ for the RCS03 (squares), TUL06 (triangles) and ROSA07 (circles) samples. In the left panel we have overplotted the correlation lines corresponding to the $L_{\mathrm{soft}} / L_{\mathrm{FIR}}$ ratios predicted by IB models for different ages and $\epsilon_{\mathrm{xeff}}$ values. The right panel shows the corresponding predictions for EB models.

For comparison, the semiempirical calibration derived by Ranalli et al. (2003) is

$$
\operatorname{SFR}\left(M_{\odot} \mathrm{yr}^{-1}\right)=1.1 \times 10^{-40} L_{\text {softX }}\left(\operatorname{erg~s}^{-1}\right)
$$

where we have adapted the original calibration to the whole FIR emission in the $1-1000 \mu \mathrm{m}$ band, to be consistent with Kennicutt (1998) (whose $L_{\text {FIR }}$ calibration is used as a proxy), and have scaled the original Kennicutt (1998) mass normalization to the range $2-120 M_{\odot}$ to be directly comparable with our results.

These calibrations should be applicable, within the range of validity shown in Fig. 1, for galaxies experiencing an extended episode of star formation at a constant star formation rate. In the case of coeval, instantaneous starbursts, the parameterization would be:

$$
\operatorname{SFS}\left(M_{\odot}\right)=2 \times 10^{-34} L_{\text {softX }}\left(\mathrm{erg} \mathrm{s}^{-1}\right)
$$

where the star formation strength $(S F S)$ is the total mass transformed into stars at the onset of the burst.

The semiempirical calibration proposed by Ranalli et al. (2003) is therefore in good agreement with the calibration derived from synthesis models for relatively unevolved star formation episodes, but it would tend to overestimate the star formation rate for galaxies that have been forming massive stars over a long time, for example tens or hundreds of millions of years.

The models do not in principle predict any dependence of the $L_{\mathrm{softX}} / L_{\mathrm{FIR}}$ ratios on the strength (i.e. total luminosity) of the star formation episodes, as proposed by some authors for the hard X-ray luminosity. Nevertheless, we want to remark that our models do not study the detailed properties of the medium surrounding the star-forming regions. The intensity of the starformation episode could, for example, have a direct effect on the dust-grain properties of the interstellar medium. $\epsilon_{\mathrm{xeff}}$ may, in addition, be a function of the burst intensity. Both effects could create a correlation between $L_{\mathrm{SoftX}} / L_{\mathrm{FIR}}$ and the star-formation burst intensity, although such a correlation is unclear in Fig. 6.

\section{Conclusions}

We have compared the $L_{\mathrm{Soft}} / L_{\mathrm{FIR}}$ values measured in a sample of 62 star-forming galaxies with the predictions by our evolutionary synthesis models, aiming to analyze the validity of semiempirical and theoretical calibrations of $L_{\mathrm{softX}}$ as a star formation rate estimator. The main results can be summarized as follows:

1. The $L_{\mathrm{softX}} / L_{\mathrm{FIR}}$ ratios are strongly dependent on the efficiency in the conversion of the mechanical energy released by the young massive starburst into soft X-ray luminosity, by interaction of the stellar winds and supernova ejecta with the surrounding interstellar medium. From theoretical predictions and observational data, we expect an $\epsilon_{\mathrm{x} e f f}$ value of few percent in starburst galaxies.

2. $L_{\mathrm{soft}} / L_{\mathrm{FIR}}$ is also dependent on the evolutionary status of the star formation episode. It increases rapidly with time during the first 5 Myr of evolution of a massive starburst, and shows a slower increase afterwards. After a (nearly) instantaneous burst of star formation, $L_{\mathrm{soft}}$ decreases slower than $L_{\mathrm{FIR}}$, as long as there remains a population of massive stars able to collapse as supernovae (up to around $35 \mathrm{Myr}$ ).

3. When star formation proceeds at a nearly constant rate during extended periods of time, the $L_{\mathrm{SoftX}} / L_{\mathrm{FIR}}$ ratio is expected to stabilize after around $40 \mathrm{Myr}$, when the number of massive stars that produce supernovae has reached an equilibrium between death and birth of new stars.

4. The $L_{\mathrm{softX}} / L_{\mathrm{FIR}}$ values measured for the sample of starforming galaxies are consistent with the predictions by the models under realistic conditions: relatively young and unevolved star formation episodes and $\epsilon_{\text {xeff }}$ values within $1-10 \%$.

5. A calibration of $L_{\mathrm{soft}}$ as a star formation rate estimator, based on the predictions of evolutionary synthesis models, has been derived. The calibration proposed by Ranalli et al. (2003) is consistent with the predictions for relatively unevolved, time-extended bursts of star formation.

Acknowledgements. J.M.M.H. and H.O. are partially funded by Spanish MEC grants AYA2004-08260-C03-03 and ESP2005-07714-C03-03. OH is funded by Spanish FPI grant BES-2006-13489. MCS acknowledges funding by Spanish M.E.C. grant AYA2004-02703, and by Spanish Ramón y Cajal fellowship El 01/08/2007.

\section{References}

Alexander, D. M., Bauer, F. E., Brandt, W.N., et al. 2003, AJ, 126, 539

Arnault, P., Kunth, D., \& Schild, H. 1989, A\&A, 224, 73

Belfort, P., Mochkovitch, R., \& Dennefeld, M. 1987, A\&A, 176, 1

Bell, E. F. 2003, ApJ, 586, 794

Calzetti, D., Armus, L., Bohlin, R. C., et al. 2000, ApJ, 533, 682

Cardelli, J. A., Clayton, G. C., \& Mathis, J. S. 1989, ApJ, 345, 245 
Cerviño, M., \& Mas-Hesse, J. M. 1994, A\&A, 284, 749

Cerviño, M., Mas-Hesse, J. M., \& Kunth, D. 2002, A\&A, 392, 19

Degioia-Eastwood, K. 1992, ApJ, 397, 542

Fabbiano, G., \& Shapley, A. 2002, ApJ, 565, 908

Gilfanov, M., Grimm, H.-J., \& Sunyaev, R. 2004, MNRAS, 351, 1365

Grimes, J. P., Heckman, T., Strickland, D., \& Ptak, A. 2005, ApJ, 628, 187

Grimm, H.-J., Gilfanov, M., \& Sunyaev, R. 2003, MNRAS, 339, 793

Helou, G., Khan, I. R., Malek, L., \& Boehmer, L. 1988, ApJS, 68, 151

Ho, L. C., Filippenko, A. V., \& Sargent, W. L. W. 1997, ApJS, 112, 315

Kennicutt R. C., Jr. 1998, ARA\&A, 36, 189

Kunth, D., Mas-Hesse, J. M., Terlevich, E., et al. 1998, A\&A, 334, 11

Leitherer, C., \& Heckman, T. M. 1995, ApJS, 96, 9

Mas-Hesse, J. M., \& Kunth, D. 1991, A\&AS, 88, 399

Mas-Hesse, J. M., \& Cerviño, M. 1999, Wolf-Rayet Phenomena in Massive Stars and Starburst Galaxies, IAUS, 193, 550

Mas-Hesse, J. M., \& Kunth, D. 1999, A\&A, 349, 765

Mezger, P. G., Smith, L. F., \& Churchwell, E. 1974, A\&A, 32, 269

Mezger, P. O. 1978, A\&A, 70, 565

Miniutti, G., Ponti, G., Dadina, M., et al. 2006, MNRAS, 373, L1
Persic, M., \& Rephaeli, Y. 2002, A\&A, 382, 843

Persic, M., \& Rephaeli, Y. 2007, A\&A, 463, 481

Persic, M., Rephaeli, Y., Braito, V., et al. 2004, A\&A, 419, 849

Ranalli, P., Comastri, A., \& Setti, G. 2003, A\&A, 399, 39 [RCS03]

Rosa-González, D., Terlevich, E., \& Terlevich, R. 2002, MNRAS, 332, 283

Rosa-González, D., Burgarella, D., Nandra, K., et al. 2007, MNRAS, 379, 357 [ROSA07]

Strickland, D. K., \& Stevens, I. R. 1999, MNRAS, 306, 43

Strickland, D. K., Heckman, T. M., Colbert, E. J. M., et al. 2004a, ApJS, 151, 193

Strickland, D. K., Heckman, T. M., Colbert, E. J. M., et al. 2004b, ApJ, 606, 829

Summers, L. K., Stevens, I. R., \& Strickland, D. K. 2001, MNRAS, 327, 385

Summers, L. K., Stevens, I. R., Strickland, D. K., \& Heckman, T. M. 2004, MNRAS, 351, 1

Takeuchi, T. T., Buat, V., Iglesias-Páramo, J., et al. 2005, A\&A, 432, 423

Tüllmann, R., Pietsch, W., Rossa, J., Breitschwerdt, D., \& Dettmar, R.-J. 2006a, A\&A, 448, 43

Tüllmann, R., Breitschwerdt, D., Rossa, J., et al. 2006b, A\&A, 457, 779 [TUL06] 